\title{
Osteonecrosis of bilateral femoral head in a post COVID-19 patient: case report
}

\author{
Ankith Chacko*, Murukan Babu, Dibin K. Thomas
}

Department of Orthopaedics, Rajagiri Hospital, Kochi, Kerala, India

Received: 21 January 2021

Accepted: 04 March 2021

\author{
*Correspondence: \\ Dr. Ankith Chacko, \\ E-mail: ankithchacko02@gmail.com
}

Copyright: (c) the author(s), publisher and licensee Medip Academy. This is an open-access article distributed under the terms of the Creative Commons Attribution Non-Commercial License, which permits unrestricted non-commercial use, distribution, and reproduction in any medium, provided the original work is properly cited.

\begin{abstract}
Osteonecrosis of the femoral head, can be secondary to various causes, which can be traumatic or atraumatic. The corticosteroid used is a common cause of non-traumatic osteonecrosis. Various viral infections have been documented to cause osteonecrosis, none of the studies report COVID-19. 23-year-old gentleman diagnosed with COVID-19. He received steroid injections as a part of treatment. Nearly after two months, he was diagnosed to have stage II osteonecrosis of both femoral heads. The cumulative dose of steroid given to the patient was not enough to consider diagnosis of steroid associated osteonecrosis of femoral heads. The combination hypercoagulability, inflammation of blood vessels, and leukocyte/platelet aggregation associated with COVID-19 might have contributed to the development of osteonecrosis in this case. So, it is also important to consider osteonecrosis as an important differential if a patient with past history of COVID-19 come with hip pain.
\end{abstract}

Keywords: Osteonecrosis of the femoral head, COVID-19, Steroid associated osteonecrosis

\section{INTRODUCTION}

Osteonecrosis of the femoral head is a progressive disease that generally affects patients in their third and fifth decades of life. Formerly it was referred to as avascular necrosis, the term osteonecrosis is now preferred. The "avascular" state of the necrotic bone is the result of disruption of blood supply to the femoral head. Osteonecrosis can be secondary to various causes, which can be traumatic or atraumatic. These causes can be fractures, dislocations, surgery, marrow fatty infiltration, alcohol, steroids, conditions predisposing to thrombophilia and many others. Meningococcemia and viral infections such as HIV, CMV, hepatitis, rubella, rubeola, varicella are already documented to cause osteonecrosis. ${ }^{1}$ In many cases a definite cause cannot be identified and conclude that group as idiopathic osteonecrosis.

There are reports of osteonecrosis in patients who have suffered SARS, most of it being associated with the use of corticosteroids in the treatment of ARDS associated with
SARS. The experience with SARS has helped in making guidelines and recommendations in the management of COVID-19. But there are no reports in literature explaining the direct association between COVID-19 and osteonecrosis of femoral head.

\section{CASE REPORT}

23-years-old gentleman diagnosed with COVID-19. He had fever, raised inflammatory markers; saturation was maintained. He received dexamethasone $6 \mathrm{mg}$ injection once daily dose for 9 days and injection methylprednisolone $40 \mathrm{mg}$ in twice daily dose for 5 days (cumulative dose is equivalent to $860 \mathrm{mg}$ of prednisolone). Patient turned COVID-19 negative subsequently. The entire disease period was clinically and symptomatically uneventful. After 56 days of recovery from COVID-19, he started feeling pain in both hips, which was a dull aching type, gradually progressing and aggravated by activities. Patient was evaluated by plain radiography and MRI as shown in Figure 1 (A-D), which showed stage II osteonecrosis of bilateral femoral heads. Risk factors of 
osteonecrosis like substance abuse, previous fractures, dislocations or surgeries around hips, chronic drug intake, and coagulation disorders were ruled out. Blood parameters were normal. The Sickling test was negative and peripheral smear showed normocytic normochromic blood picture. Haemoglobin electrophoresis was done to rule out beta- thalassaemia and other hemoglobinopathies
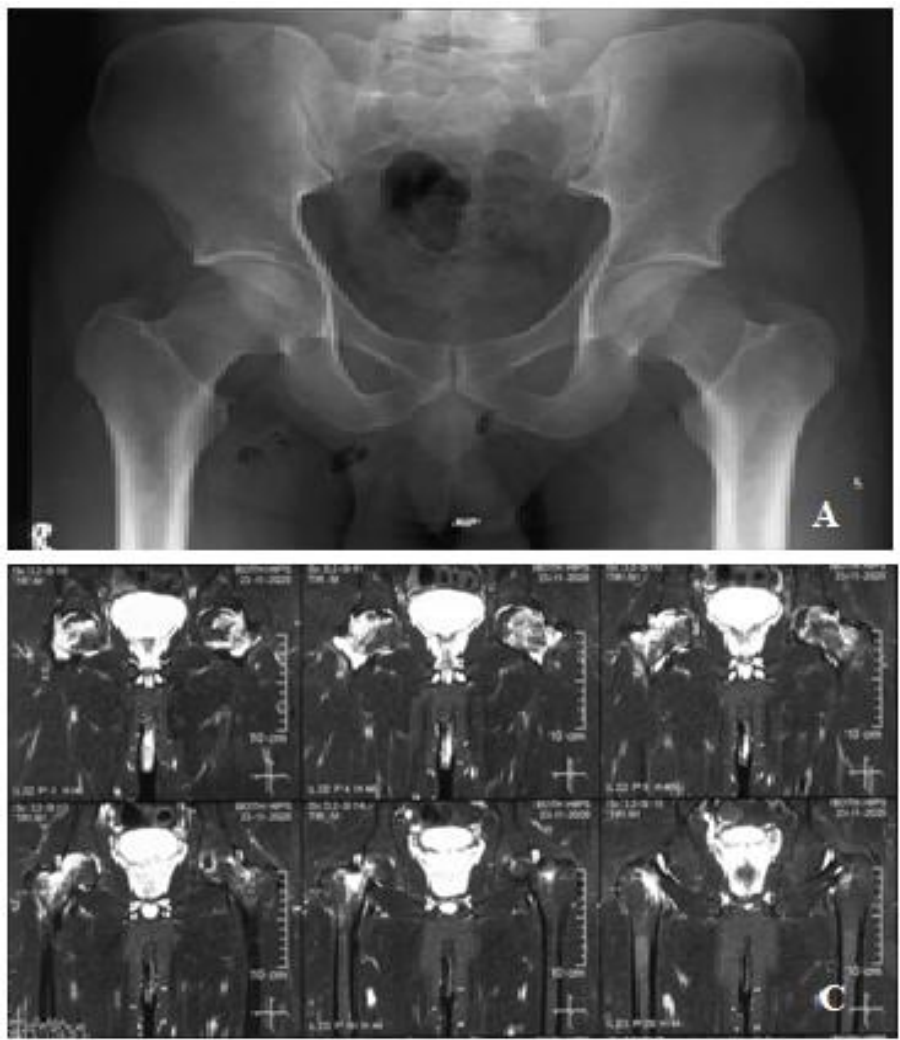

and was normal. Erythropoietin level was also normal. Blood cultures were negative.

The patient was managed by core decompression of both femoral heads and BMAC (bone marrow aspirate concentrate) injection. Figure 2 shows the immediate postoperative radiograph.
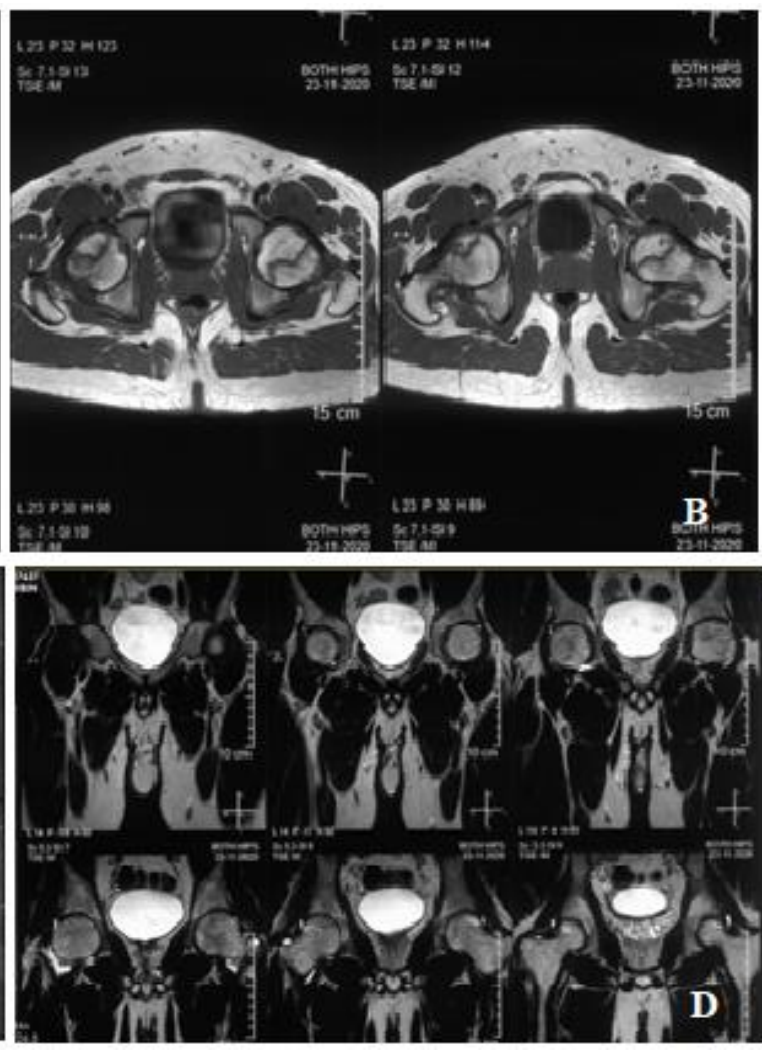

Figure 1: (A-D) Plain radiography and MRI of the patient showing signal intensity changes in both femoral heads in geographical pattern, no collapse of femoral heads and congruity of both hip joints maintained, suggestive of FICAT and ARLET stage II osteonecrosis of both femoral heads.

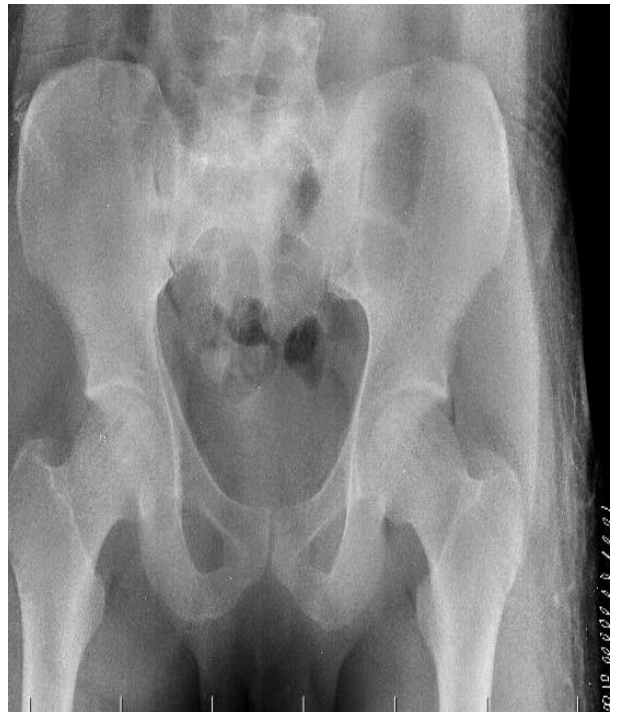

Figure 2: Immediate postoperative radiograph after core decompression and BMAC injection.

\section{DISCUSSION}

Many theories are postulated regarding the pathogenesis of osteonecrosis, but none of them can fully account for the wide variety of attributed causes. Hypotheses include ischemia from mechanisms like direct cellular toxicity, and alteration of differentiation of mesenchymal stem cells. Femoral heads function as closed compartments and some of these incidents may cause an increase in intraosseous bone marrow pressure. This may lead to a pressure in the venule and capillary system and ultimately leads to decrease in blood flow to femoral head and results in ischemia, and osteonecrosis.

Reports state that there are 15,000 to 30,000 cases of osteonecrosis of femoral heads diagnosed per year in the USA. But for India there are no statistical data available at present. Considering the population size of India, probably 70,000 to 90,000 new patients per year get affected with osteonecrosis of femoral head. ${ }^{2}$ The disease is more frequently seen in men than in women. 


\section{Steroid and osteonecrosis}

The most common cause of non-traumatic osteonecrosis of femoral head is corticosteroid use. ${ }^{3}$ In spite of recent advances, the exact pathogenetic mechanism of corticosteroid associated osteonecrosis remains unclear. Pathogenesis is probably multifactorial involving various genetic and environmental factors. But eventually the changes that occur results in failure to deliver the nutrients to the femoral head. These events in the early stages may include vascular damage, mechanical stresses, increased intraosseous pressure, adipocyte dysfunction, defects in apoptosis and coagulation dysfunction. But the end result is the same, the failure of delivering nutrients to watershed areas of the femoral head and necrosis of osteocytes.

The risk of corticosteroids on the development of osteonecrosis is dependent on daily dose, cumulative dose, maximum dose and route of administration, as well as underlying disease states, patient demographics and many others.

Koo et al attempted to determine the cumulative dose and average duration of steroids required to cause osteonecrosis of femoral heads. ${ }^{4}$ Patients who were diagnosed at the early stage by magnetic resonance imaging (MRI) without positive findings on plain radiographs were selected. The total dose of steroid, which was used until the time of detection of osteonecrosis by MRI, ranged from 1800 to $15505 \mathrm{mg}$ prednisolone or its equivalent (mean $5928 \mathrm{mg}$ ). The time taken for the diagnosis by MRI ranged from 1 month to 16 months (mean 5.3 months) from the start of steroid treatment.

In another study, Powell et al concluded that a cumulative dose of 480-4320 mg of dexamethasone (or its equivalent dose) will be a risk factor for osteonecrosis. ${ }^{1}$

Chan et al studied Steroid-induced osteonecrosis in 71 patients with severe acute respiratory syndrome (SARS) in 2003 (Hong Kong). ${ }^{5}$ Seven patients developed radiological evidence of osteonecrosis. The prevalence of osteonecrosis in the cohort was $9.9 \%$. A total corticosteroid dosage of $>2000 \quad \mathrm{mg}$ methylprednisolone, $>1900 \mathrm{mg}$ hydrocortisone, $>13340 \mathrm{mg}$ hydrocortisone-equivalent corticosteroid therapy, and $>18$ days on corticosteroid therapy were found to be significant risk factors for the subsequent development of osteonecrosis.

In 2017, Association Research Circulation Osseous (ARCO) proposed criteria for diagnosing GlucocorticoidAssociated Osteonecrosis of Femoral Heads. ${ }^{6}$ Criteria by ARCO includes; (i) patients should have a history of glucocorticoid use of $>2 \mathrm{~g}$ of prednisolone or its equivalent within a 3-month period; (ii) osteonecrosis should be diagnosed within 2 years after glucocorticoid usage, and (iii) patients should not have other risk factor(s) besides glucocorticoids. These criteria should be satisfied before marking glucocorticoid as the etiological agent for the osteonecrosis of femoral heads.

The cumulative dose steroid taken by the reported patient was $54 \mathrm{mg}$ of dexamethasone and $400 \mathrm{mg}$ of methylprednisolone over 2 weeks duration, which is equivalent to $860 \mathrm{mg}$ of prednisolone, $3440 \mathrm{mg}$ of hydrocortisone or $688 \mathrm{mg}$ methylprednisolone. ${ }^{1} \mathrm{~A}$ cumulative dose of $860 \mathrm{mg}$ of prednisolone is not satisfying the ARCO criteria for diagnosing as steroid associated osteonecrosis of femoral heads. ${ }^{6}$ The observations by Chan et al and Koo et al, is also not supportive for the diagnosis of steroid associated osteonecrosis of femoral heads in this case. $^{4,5}$

At this stage we have to think about the role of COVID-19 caused by the novel corona virus itself in development of osteonecrosis of femoral heads if any.

\section{COVID-19 and osteonecrosis}

Corona virus 2019 disease COVID -19, is caused by the severe acute respiratory syndrome corona virus 2 (SARSCoV-2). Even Though it primarily affects the respiratory system, COVID -19 has multiple organ system involvement including the musculoskeletal system. Epidemiological data regarding SARS (2002-2004) reported various musculoskeletal problems like myalgia, arthralgia, osteoporosis, and osteonecrosis as common complications. The rate of occurrence of osteonecrosis was 5 to $58 \%$ in SARS. The majority of cases affect the femoral heads, femoral condyles, talus, calcaneus and humeral heads. ${ }^{7-9}$

There are many reports stating that COVID -19 and SARS are hypercoagulable states and they generate a significantly increased risk for thrombosis. ${ }^{10,11}$ In SARSCoV-1 infection the leukocyte aggregation and blood vessel inflammation has association with expression of the E3 ubiquitin ligase gene TRIM55 in vascular smooth muscle cells. ${ }^{12}$ The relevance of coagulation abnormalities in COVID-19 are becoming increasingly clear as a substantial proportion of patients develop, sometimes recognized, venous and arterial thromboembolic events. ${ }^{13}$ COVID-19 may predispose to increased incidence of both arterial and venous thrombotic events and the risk factors for the same might be excessive inflammation, hypoxia, immobilization or diffuse intravascular coagulation. Acute pulmonary embolism (PE), deep-vein thrombosis, ischemic stroke, myocardial infarction or systemic arterial embolism have been reported among COVID-19 patients. $^{14}$

The incidence of arterial thrombosis among patients with COVID-19 has an incidence rate of 5 to $10 \% .{ }^{15,16}$ Although many questions regarding the pathogenesis of osteonecrosis of femoral heads remain unanswered, a great number of published series are in favour of the theory of increased intravascular coagulation. ${ }^{15}$ 
In some patients with COVID-19 infection, the body responds with exuberant cytokine storm. Immunological studies have confirmed that pro-inflammatory cytokines like interleukin 6 (IL-6), IL-17A, and tumor necrosis factor $\alpha$ were elevated in the majority of patients with severe infection with COVID-19. ${ }^{17,18}$ Hypercoagulability is a usual finding in inflammation. Pro-inflammatory cytokines have a role in the platelet aggregation and intravascular clot formation and are also involved in the down regulation of physiological anticoagulant pathways in the body. ${ }^{19}$

The combination hypercoagulability, inflammation of blood vessels, and leukocyte/platelet aggregation may impair microvascular blood flow, which subsequently can lead to development of osteonecrosis. This hypercoagulability associated with COVID-19 could have implications in the pathogenesis of osteonecrosis of femoral heads in the reported case.

Mehta et al has suggested the judicious use of glucocorticoids in patients with COVID-19 considering the risk for osteonecrosis. ${ }^{20} \mathrm{We}$ couldn't find any literature marking the COVID-19 among the primary causes of osteonecrosis of femoral heads and there are no reports explaining the pathogenesis of COVID-19 leading to osteonecrosis, other than the steroid associated osteonecrosis of femoral head among COVID-19 patients. ${ }^{21}$ The patient has not taken the sufficient dose of steroid for considering the possibility of corticosteroid associated osteonecrosis of femoral heads, so, COVID-19 and its hypercoagulable state itself might be led to osteonecrosis in this reported case.

\section{CONCLUSION}

Despite the worldwide occurrence, the pathology, complications and sequelae of COVID-19 has not been fully established. The various treatment protocols proposed has been met with controversies. More studies are yet to come before the final verdict. Corticosteroids are widely used in the management of COVID-19 even in the absence of a solid proof for the efficacy of the same. Though the experience with corticosteroids in the previous SARS epidemic cautions about the possible incidence of osteonecrosis, reports about the same in COVID-19 is not abundant. Occurrence of osteonecrosis in the reported case even with a lower dose of corticosteroids calls for discussions about the hypercoagulable state and increased risk of osteonecrosis of femoral head in COVID-19 cases. It is also important to consider osteonecrosis as an important differential if a patient with past history of COVID-19 come with hip pain. The empirical use of even low dose steroids in the management of COVID-19 should be reconsidered. Further research regarding the hypercoagulable state and the association of osteonecrosis in COVID-19 has to be made.
Funding: No funding sources Conflict of interest: None declared

Ethical approval: Not required

\section{REFERENCES}

1. Powell C, Chang C, Naguwa SM, Cheema G, Gershwin ME. Steroid induced osteonecrosis: An analysis of steroid dosing risk. Autoimmun Rev. 2010;9(11):721-43.

2. Petek D, Hannouche D, Suva D. Osteonecrosis of the femoral head: pathophysiology and current concepts of treatment. EFORT Open Rev. 2019;4(3):85-97.

3. Seamon J, Keller T, Saleh J, Cui Q. The Pathogenesis of Nontraumatic Osteonecrosis. Arthritis. 2012:1-11.

4. Koo KH, Kim R, Kim YS, Ahn IO, Cho SH, Song $\mathrm{HR}$, et al. Risk Period for Developing Osteonecrosis of the Femoral Head in Patients on Steroid Treatment. Clin Rheumatol. 2002;21(4):299-303.

5. Chan MHM, Chan PKS, Griffith JF, Chan IHS, Lit LCW, Wong CK, et al. Steroid-induced osteonecrosis in severe acute respiratory syndrome: a retrospective analysis of biochemical markers of bone metabolism and corticosteroid therapy. Pathology. 2006;38(3):229-35.

6. Yoon BH, Jones LC, Chen CH, Cheng EY, Cui Q, Drescher W, et al. Etiologic Classification Criteria of ARCO on Femoral Head Osteonecrosis Part 1: Glucocorticoid-Associated Osteonecrosis. J Arthroplasty. 2019;34(1):163-168.

7. Disser NP, De Micheli AJ, Schonk MM, Konnaris MA, Piacentini AN, Edon DL, et al. Musculoskeletal Consequences of COVID-19. J Bone Jt Surg. 2020 Jul 15;102(14):1197-204.

8. Hong N, Du XK. Avascular necrosis of bone in severe acute respiratory syndrome. Clin Radiol. 2004;59(7):602-8.

9. Griffith J. Musculoskeletal Complications of Severe Acute Respiratory Syndrome. Semin Musculoskelet Radiol. 2011;15(5):554-60.

10. Abou-Ismail MY, Diamond A, Kapoor S, Arafah Y, Nayak L. The hypercoagulable state in COVID-19: Incidence, pathophysiology, and management. Thromb Res. 2020;194:101-15.

11. Maier CL, Truong AD, Auld SC, Polly DM, Tanksley C-L, Duncan A. COVID-19-associated hyperviscosity: a link between inflammation and thrombophilia? The Lancet. 2020;395(10239):17589.

12. Gralinski LE, Ferris MT, Aylor DL, Whitmore AC, Green R, Frieman MB, et al. Genome Wide Identification of SARS-CoV Susceptibility Loci Using the Collaborative Cross. Panthier J-J, editor. PLOS Genet. 2015;11(10):1005504.

13. Levi M, Thachil J, Iba T, Levy JH. Coagulation abnormalities and thrombosis in patients with COVID-19. Lancet Haematol. 2020;7(6):438-40.

14. Klok FA, Kruip MJHA, van der Meer NJM, Arbous MS, Gommers DAMPJ, Kant KM, et al. Incidence of thrombotic complications in critically ill ICU 
patients with COVID-19. Thromb Res. 2020;191:145-7.

15. Lykissas MG, Kostas-Agnantis IP, Gelalis ID, Vozonelos G, Korompilias AV. The role of hypercoagulability in the development of osteonecrosis of the femoral head. Orthop Rev. 2012;4(2):17.

16. Bilaloglu S, Aphinyanaphongs Y, Jones S, Iturrate E, Hochman J, Berger JS. Thrombosis in Hospitalized Patients With COVID-19 in a New York City Health System. JAMA. 2020;324(8):799.

17. Liu Y, Yang Y, Zhang C, Huang F, Wang F, Yuan J, et al. Clinical and biochemical indexes from 2019nCoV infected patients linked to viral loads and lung injury.Sci China Life Sci. 2020;63(3):364-74.

18. Gralinski LE, Sheahan TP, Morrison TE, Menachery VD, Jensen K, Leist SR, et al. Complement
Activation Contributes to Severe Acute Respiratory Syndrome Coronavirus Pathogenesis. Subbarao K, editor. mBio. 2018;9(5):01753-18.

19. Dosquet C, Weill D, Wautier JL. Cytokines and thrombosis. J Cardiovasc Pharmacol. 1995;25(2S):13-9.

20. Mehta N, Mazer-Amirshahi M, Alkindi N, Pourmand A. Pharmacotherapy in COVID-19; A narrative review for emergency providers. Am J Emerg Med. 2020;38(7):1488-93.

21. Zhang B, Zhang S. Corticosteroid-Induced Osteonecrosis in COVID-19: A Call For Caution. J Bone Miner Res. 2020;35(9):1828-9.

Cite this article as: Chacko A, Babu M, Thomas DK. Osteonecrosis of bilateral femoral head in a post COVID-19 patient: case report. Int J Res Orthop 2021;7:674-8. 